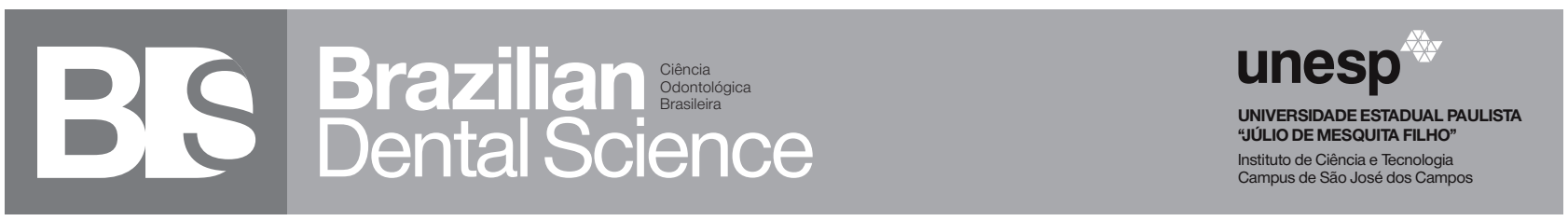

\title{
EDITORIAL
}

\section{BDS IN THE CONTEXT OF ACHIEVEMENTS}

Impact Factor (IF) means the science-merit index that represents the average annual number of citations that manuscripts published in a specific journal received in the last two or three years [1, $2]$. It expresses the relative importance of the journal in the field from the view of the scientific community.

Different databases have their own impact factor calculation based on the citations in the indexed journals.

For the journals indexed on the Web of Science, those are ranked by JCR (Journal Scientific Report)/ISI (Institute for Science and Information) in JIF (Journal Impact Factor). For Scopus by SJR (Scimago Journal Rank-citescore)/SNIP (Source Normalized Impact per Paper); etc.

Since we got Scopus indexation in 2017, BDS has been growing its CiteScore index from 0.3 to 1.0 in 2020 [3]. This bibliometric index shows a positive trend for BDS during the last years. Our team is focused on reaching a score higher than "1.0".

Furthermore, BDS has improved its Editorial Board. Twenty-four section editors and 30 assistant editors are weekly directly involved with the process of the manuscripts submitted to the journal. Opportunities arising from the pandemic brought improvements in the speed of evaluation process, distribution of the steps, partnership, digital or remote meetings, and visibility of the journal.

The communion of ideals among the team members led BDS to a high profile on social networks as Instagram, Facebook and LinkedIn. Our posts have been shared with researchers with great success.

By 2021, authors and readers can expect issues with the highest quality in science, committed to innovation, and sharing.

Welcome to BDS journal once again.

\section{REFERENCES}

1. Frankernberger, R., Van Meerbeek, B. JAD in the context of bibliometric data. $\operatorname{JAD}(2020)$, n5, p. 439. doi:10.3290/j.jad.a45410

2. Fernandes V., Salviano LR. Indicadores JCR, SNIP,SJRe Google Scholar. http:// portal.utfpredu.br/pesquisa-e-pos-graduacao/indicadores/indicadoresexternos/indicadores-externos

3. CiteScoreTracker. Scopus. [Internet]. Access in:16 Dec 2020. Avaiable at: https://www.scopus.com/sourceid/21100831445

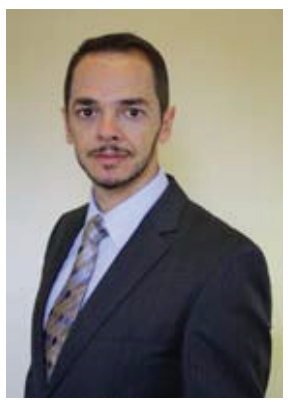

Full Professor

Sérgio Eduardo de Paiva Gonçalves Editor-in-Chief Institute of Science and Technology São Paulo State University (Unesp) 


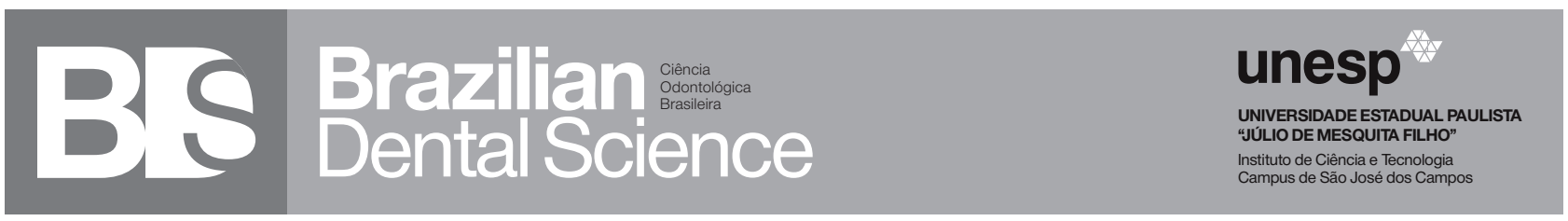

\section{EDITORIAL}

\section{BDS NO CONTEXTO DE REALIZAÇÕES}

Fator de impacto (FI) significa o índice científicométrico que representa a média anual de citações que os manuscritos publicados em um determinado periódico receberam nos últimos dois ou três anos [1,2]. Expressa a importância relativa da revista na área do ponto de vista da comunidade científica.

Diferentes bancos de dados têm seu próprio cálculo de fator de impacto com base nas citações nos periódicos indexados.

Para os periódicos indexados no Web of Science, eles são classificados pelo JCR (Journal Scientific Report) / ISI (Institute for Science and Information) no JIF (Journal Impact Factor). Para Scopus por SJR (Scimago Journal Rank-citescore) / SNIP (Source Normalized Impact per Paper); etc.

Desde que obtivemos a indexação Scopus em 2017, a BDS vem crescendo seu índice CiteScore, aumentando de 0,3 para 1,0 em 2020 [3]. Este índice bibliométrico mostra uma tendência positiva para o BDS nos últimos anos. Nossa equipe está focada no futuro com uma pontuação superior a "1.0".

Além disso, a BDS aprimorou seu Conselho Editorial. Vinte e quatro editores de seção e 30 editores assistentes estão diretamente envolvidos semanalmente com o processo dos manuscritos submetidos à revista. As oportunidades decorrentes da pandemia trouxeram melhorias na agilidade do processo de avaliação, na distribuição das etapas, na parceria, na realização de reuniões digitais ou remotas e visibilidade do periódico.

A comunhão de ideais entre os membros da equipe levou a BDS a um destaque nas redes sociais como Instagram, Facebook e LinkedIn. Nossas postagens foram compartilhadas pelos pesquisadores com grande sucesso.

Em 2021, autores e leitores podem esperar edições com a mais alta qualidade em ciência, compromisso com a inovação e divulgação.

Bem-vindo ao jornal BDS mais uma vez.

\section{REFERÊNCIAS}

1. Frankernberger, R., Van Meerbeek, B. JAD in the context of bibliometric data. $\operatorname{JAD}(2020)$, n5, p. 439. doi:10.3290/j.jad.a45410

2. Fernandes V., Salviano LR. Indicadores JCR, SNIP,SJRe Google Scholar. http:// portal.utfpredu.br/pesquisa-e-pos-graduacao/indicadores/indicadoresexternos/indicadores-externos

3. CiteScoreTracker. Scopus. [Internet]. Access in: 16 Dec 2020. Avaiable at: https://www.scopus.com/sourceid/21100831445

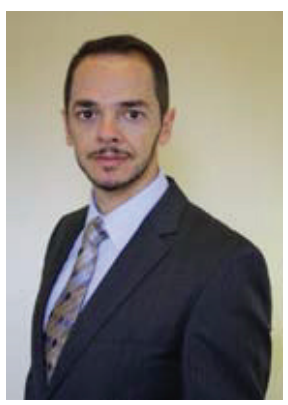

Professor titular

Sérgio Eduardo de

Paiva Gonçalves

Editor Chefe

Instituto de Ciencia e

Tecnologia

Universidade Estadual

Paulista - Unesp 